\title{
IDENTIFICATION OF MICRORNAS IN 12 PLANT SPECIES OF FABACEAE
}

\author{
Abdul Ghani', Muhammad Din*1, Iftekhar Ahmed Baloch ${ }^{1}$, Muhammad Younas Khan Barozai ${ }^{1}$ \\ ${ }^{1}$ Department of Botany, University of Balochistan, Sariab Road Quetta, Pakistan \\ * Corresponding author E-mail deen.2006@yahoo.com \\ Phone \#+92-0333-7817319 Fax +92-081-9211-277
}

\begin{abstract}
MicroRNAs (miRNAs) are tiny, non-coding and regulatory RNAs approximately 21 nucleotides in length. They are reported in various plants but still needs discovery in important plant species. The 12 plant species of Fabaceae were subjected this time to identify their miRNAs. The comparative genomics approaches with combination of various bioinformatics' tools were applied to find the novel miRNAs. This research leads to the finding of 29 miRNAs belonging to 13 miRNA families. From the 29 miRNAs, nine belongs to Arachis duranensis, four to Lotus japonicas, three to each Pisum sativum and Arachis hypogaea, two to each Arachis ipaensis and Phaseolus vulgaris and one to each Cicer arietinum, Phaseolus acutifolius, Lupinus luteus, Glycyrrhiza uralensis, Robinia pseudoacacia and Lathyrus odoratus. These findings will be useful in the future to design and develop desirable traits in the 12 plant species of Fabaceae.

Keywords: Comparative genomics; Fabaceae; MicroRNAs

\section{Introduction}

MicroRNAs (miRNAs) are endogenous, small RNAs, 21 nt long and are found in a large number of animals and plants, such as Caenorhabditis elegans, Oryza sativa, Arabidopsis thaliana, Cotton, Mouse and human beings [1]. They are found in large number of non-coding RNAs [2], and play a central role in post-transcriptional gene regulation by degrading or by repressing target messenger RNAs in eukaryotes [3]. Since the discovery of the first miRNA, lin-4, in Caenorhabditis elegans [4],

thousands of miRNAs has been identified by experimental cloning or computational approaches [5, 6].

In plants, miRNA genes are transcribed by RNA polymerase II, and primary miRNA transcripts are subsequently capped, spliced and poly-adenylated [7]. Plant miRNAs are processed endogenously while the mature miRNAs are transferred into cytoplasm with the help of HASTY, a miRNA transporter [8]. In plants, miRNAs are involved in various features of plant growth and development, including leaf
\end{abstract}


morphology and polarity, lateral root formation, hormone signaling, transition from immature to adult vegetative stage and vegetative to flowering phase, flowering spell, floral organ identity and reproduction [9].

The miRNAs are also taking part in plant stress responses. A number of miRNAs are regulated in response to different stress conditions, which suggests that miRNA-directed post-transcriptional regulation of their respective target genes is important to cope with the stress conditions [10]. Plant miRNAs are conserved in nature which provides us a good basis for the identification of novel miRNAs in a number of plant species $[\mathbf{1 1}, \mathbf{1 2}]$. In this research paper we identified total of 29 newly miRNAs (out of 13 miRNAs families; 156, 159, 160, $162,164,166,167,168,390,393,394,5630$ and 5658) in 12 diverse plant species (viz; Cicer arietinum, Arachis duranensis, Phaseolus acutifolius, Pisum sativum, Lotus japonicas, Arachis hypogaea, Lupinus luteus, Phaseolus vulgaris, Arachis ipaensis, Glycyrrhiza uralensis, Robinia pseudoacacia, Lathyrus odoratus) of family Fabaceae, using all publicly available ESTs (Expressed Sequence Tags) subjected for the computational identification methodology of miRNAs orthologue. These findings will be useful for tracing the evolution of micro
RNAs by examining their expression in common ancestors of different plant species.

\section{Materials and Methods}

\section{Retrieval of miRNAs Through Similarity Search}

The similar approach as adopted previously [11] was practiced to predict the novel miRNAs in family Fabaceae. We subjected plant precursors miRNAs (pre-miRNAs) sequences, retrieved from the microRNA Registry Database (Version Rfam 18 released on November 3,2011$)$ [13], compared to the publically available Fabaceae ESTs from the dbEST (http://www.ncbi.nlm.nih.gov/dbEST/dbEST_summa ry.html) released on (1 ${ }^{\text {st }}$ March, 2012) at http://blast.ncbi.nlm.nih.gov/Blast.cgi by the use of blastn [14]. The basic local alignment search tool (BLAST) parameter settings were adjusted, as follows: expect values $\sim 1,000$; low density was chosen as the sequence filter, database (others Fabaceae) program selection (somewhat similar sequence) and all other parameters were used as default. The consequence results in FASTA formats of all the primary potential candidates' pre-miRNA sequences having maximum 4 mis-matches were saved. The frequented ESTs from the same gene were taken away and produced a single tone EST using blastn with default parameters. For the confirmation that novel miRNAs as non-protein coding RNAs, the 
Pure Appl. Bio., 2(3): 104-115, Sep- 2013.

initial potential candidates' pre-miRNA sequences were subjected to BALST against protein database at National Center for Biotechnology Information (NCBI) using blastx (http://blast.ncbi.nlm.nih.gov/Blast.cgi?PROGRAM= blastx\&BLAST_PROGRAMS) with default parameter to eliminate the protein coding RNAs [15].

\section{Prediction of Hairpin Structures}

To retrieve the secondary structure of the initial candidate's sequences we used the Zuker folding algorithm with MFOLD (version 3.5) [16], publicly available at http://www.bioinfo. rpi.edu/applications/mfold/rna/form1.cgi. Default parameters were used; RNA sequence (linear), folding temperature $\left(37^{\circ} \mathrm{C}\right)$, ionic condition $(1 \mathrm{M}$ $\mathrm{NaCl}$ with no divalent ions), percent sub-optimality number (5); maximum interior/ bulges loop size (30), and all other with preset values. The maximum (- $\log )$ free energy structures were chosen on the basis of trial and error, as termed by Barozai et al. [17]. The threshold values used to select a miRNA were same as described by Zhang et al. [18]. The stem portion of the hairpin checked for the mature sequences with at least 12 base pairs involved in Watson-Crick or $\mathrm{G} / \mathrm{U}$ base pairing between the mature miRNA and the opposite strand (miRNA*).

\section{Filtration of Sequence and Structural Features}

For the filtration of sequence and structural features, calculation was made of the GC content, core minimum free energy (mfe), hairpin mfe and core hairpin ratio (ch_ratio) as determined by Li et al. [19]. A small modification for core mfe calculation, was used by Barozai et al. [11]. Drosha, a RNA-III like endonuclease, recognizes and cleaves the primary transcripts of mature miRNAs (pri-miRNA) at 22 nucleotides upstream from the position of the first nucleotide (Pfn - 22) and the 24 nucleotides downstream the terminal nucleotide $(\operatorname{Ptn}+24)$ to yield pre-miRNA in animals [19]. In plant premiRNAs, the mature miRNA sequences were located away, so, the Pfn - 24 and Ptn +26 were included in the core portion of the hairpin structure. The mfe for core and secondary structures were calculated by MFOLD (version 3.5) [16], publicly available at http://www.bioinfo.rpi.edu/applications/mfold/rna/for ml.cgi. The parameters were adjusted same as reported earlier. For ch_ratio calculation, we divided the core mfe by the hairpin mfe, and the quotient is denoted as the ch_ratio.

\section{Conservation and Phylogenetic Analysis of}

\section{Fabaceae miRNAs}

As the miRNAs are conserved in nature among 
eukaryotes; subsequently we made conservation and phylogenetic study of the Fabaceae miRNAs with others plant miRNAs. The conservation and phylogenetic study of miRNAs were prepared by fetching the FASTA format of pre-miRNAs sequences for Arabidopsis thaliana (ath), rice (Oryza sativa, osa) and Populus trichocarpa (Ptc), from the microRNA Registry Database (Version Rfam 18.0 released November $\left.3^{\text {rd }}, 2011\right)$ [13] and aligned with Fabaceae miRNAs using the publically available weblogo [20] and ClustalW to create cladogram tree [21] respectively. The results were saved.

\section{Prediction of Fabaceae miRNA Targets}

To predict the Fabaceae miRNAs targets, we used the NCBI blastn program [14], by performing the mature miRNA sequences as queries [22]. The parameters were adjusted as, Database; Nucleotide collection (nr/nt), organism; Fabaceae (taxid: 3803) and Program Selection; highly similar sequences (megablast). The sequences showing $80 \%$ query coverage were selected and giving for the target validation to RNA-hybrid submission algorithm publically available at (http://bibiserv.techfak.unibielefeld.de/rnahybrid/submission.html), a miRNAs target prediction tool [23] for the confirmation of the targets. The results validated by RNAhybrid were saved.

\section{RESULTS AND DISCUSSION}

\section{The Newly identified Fabaceae miRNAs}

Data mining, homology search and comparative genomics resulted many putative and interesting findings in various plant species $[24,25]$. Publically available ESTs were computationally considered for the prediction of novel miRNAs in family Fabaceae. Twenty nine (29) putative pre-miRNAs of Fabaceae were identified by similarity search followed by the filtration and completion strategy. The 29 newly miRNAs belong to 13 miRNA families from the known Fabaceae ESTs (Figure\#1). The 13 miRNA families (miR-156, 159, 160, 162, 164, 166, 167, 168, $390,393,394,5630$ and 5658) are observed for the first time in Fabaceae. The species were observed in; Arachis duranensis miR-156, 162, 166(a,b), 168(a,b), 390, Lotus japonicas miR-160(a,b), 162(a,b), Arachis hypogaea miR-162, 166(a,b,c), Pisum sativum miR159, 160, 164, Phaseolus acutifolius miR-159, 5658, Phaseolus vulgaris miR-167, 168, Arachis ipaensis miR-162, 390, Glycyrrhiza uralensis miR-393, Cicer arietinum miR-156, Lupinus luteus miR-162, Robinia pseudoacacia miR-394, Lathyrus odoratus miR5630. All the novel Fabaceae miRNAs considered as a valid candidates after satisfying the validation formula for biogenesis and expression of the miRNAs, given by Ambros et al. [26]. The novel Fabaceae miRNAs fulfilled the criteria B, C and D. 
According to Ambros et al. [26] only the criterion D is enough for homologous sequences to validate as new miRNAs in different species.

\section{Characterization of the Fabaceae miRNAs}

The Table \#1 characterizes the novel Fabaceae miRNAs in terms of reference miRNA families, accession numbers, plant names, mature sequences, minimum free energies (MFEs), mature sequence arms, number of miss-matches, total scores, query courage, expected values, maximum identities percentage $(\%)$, mature sequence lengths, GC content $\%$ and precursor length. MFEs play a crucial role in the prediction of stem-loop structure. The newly identified Fabaceae pre-miRNAs’ MFEs ranges from $-12.30 \mathrm{kcal} \mathrm{mol}^{-1}$ to -87.70 with in an average of $\sim$ $39.95 \mathrm{kcal} \mathrm{mol}^{-1}$. The majority $55.17 \%$ (16 out of 29 ) Fabaceae pre-miRNAs showed mature sequences at $5^{\prime}$ arm, and the remaining $44.82 \%$ (13 out of 29 ) premiRNAs have 3' arm sequences as illustrated in (Figure\#1). The novel identified Fabaceae majority miRNAs, $69 \%$ miRNAs (20 out of 29 ) are perfectly $(100 \%)$ matched with their corresponding reference miRNAs, whereas $27 \%$ ( 8 out of 29 ) showed one nt mismatched and the remaining $3.44 \%$ (1 out of 29$)$ have a difference of two nt from their homologs. Similar results have been reported in different organisms by Reinhart et al. [27] and Barozai et al. [11]. Total score of the pre-miRNAs were showed, maximum were 141 and minimum were 46.4 with an average of 98.18. Query coverage of the pre-miRNAs was calculated with the maximum value $100 \%$ and minimum value $54 \%$ with an average of $86 \%$. Expect value and maximum identities were also presented in (Table\#1). Three (out of 29) mature miRNA sequences consist of 20 nucleotides (nts), 25 consist of $21 \mathrm{nts}$ and one consists of 22 nts. Majority of the mature sequences 17 (out of 29) consist of 51-66\% GC content and 12 (out of 29) consist of 33-50\% GC content. The Fabaceae pre-miRNA length range from 60 to $180 \mathrm{nt}$ with an average of $100 \mathrm{nt}$ Maximum, 15 out of 29 pre-miRNAs have a sequence length range of $60-90 \mathrm{nt}$, following by 9 from 91-120 and 5 from 121-180 nt sequence length range. All the mature sequences of Fabaceae miRNAs are in the stem regions of the stem-loop structures, as shown in (Figure\#1). These findings are same as described by many researchers $[18, \mathbf{1 1}, \mathbf{2 8}$, 29]. The predicted miRNA stem-loop structures showed that 14 mature sequences are $100 \%$ hybridized and the remaining 15 mature sequences have (71-95\%) hybridization with the opposite arms (miRNAs*) in the stem region and the hairpin precursors do not contain large internal loops or bulges (Figure\#1). Similar results were given for the different organisms' miRNAs $[13, \mathbf{1 7}, \mathbf{2 9}]$. The 
Table \# 1: Fabaceae miRNAs characterization in terms of SM, source miRNAs, Acc. \# accession number, PN plant name, MS mature sequence, MFE minimum free energy, MSA mature sequence arm, NM number of mismatches (showed in color and enlarged font size), TS total score, QC query coverage, EV expect value, MI maximum identities, MSL mature sequence length, GC\% guanine and cytosine percentage and PL precursor miRNA length.

\begin{tabular}{|c|c|c|c|c|c|c|c|c|c|c|c|c|c|c|}
\hline $\begin{array}{l}\text { Fabaceae } \\
\text { MiRNAs }\end{array}$ & SM & Acc. \# & PN & MS & MFE & MSA & NM & TS & QC\% & EV & $\begin{array}{c}\text { MI } \\
\%\end{array}$ & MSL & $\begin{array}{c}\mathrm{GC} \\
\%\end{array}$ & PL \\
\hline car-miR156 & ath-miR156e & HO063979 & Cicer arietinum & TGACAGAAGAGAGTGAGCAC & -52.50 & 5 , & 0 & 104 & $99 \%$ & $5 e-21$ & 81 & 20 & 50 & 88 \\
\hline adu-miR156 & ath-miR156h & GW954185 & Arachis duranensis & TGACAGAAGAATAGAGAGCAC & -41.30 & 5 , & 1 & 116 & $94 \%$ & $2 \mathrm{e}-13$ & 90 & 20 & 45 & 98 \\
\hline pac-miR159 & ath-miR159b & HO805845 & Phaseolus acutifolius & TTTGGATTGAAGGGAGCTCTA & -87.70 & 3 , & 1 & 69.8 & $94 \%$ & 1e-10 & 69 & 21 & 42 & 180 \\
\hline psa-miR159 & ath-miR159b & FG534720 & Pisum sativum & TT TGGATTGAAGGGAGCTCTA & -21.70 & 3 & 1 & 89.1 & $55 \%$ & $1 \mathrm{e}-04$ & 95 & 21 & 42 & 78 \\
\hline lja-miR160a & ath-miR160a & DC595011 & Lotus japonicus & TGCCTGGCTCCCTGTATGCCA & -51.90 & 5 , & 0 & 53.6 & $97 \%$ & $8 \mathrm{e}-06$ & 76 & 21 & 61 & 83 \\
\hline lja-miR160b & ath-miR160a & FS344717 & Lotus japonicus & TGCCTGGCTCCCTGTATGCCA & -48.70 & 5 , & 0 & 93.3 & $96 \%$ & $9 e-18$ & 85 & 21 & 61 & 83 \\
\hline psa-miR160 & ath-miR160a & FG538087 & Pisum sativum & TGCCTGGCTCCCTGTATGCCA & -53.30 & 5 , & 0 & 75.2 & $96 \%$ & $3 e-12$ & 80 & 21 & 61 & 83 \\
\hline adu-miR162 & ath-miR162b & GW949510 & Arachis duranensis & TCGATAAACCTCTGCATCCAG & -31.90 & 3 , & 0 & 93.3 & $90 \%$ & $9 e-18$ & 81 & 21 & 47 & 90 \\
\hline ahy-miR162 & ath-miR162b & GO323824 & Arachis hypogaea & TCGATAAACCTCTGCATCCAG & -35.90 & 3 & 0 & 98.7 & $90 \%$ & $2 \mathrm{e}-19$ & 82 & 21 & 47 & 90 \\
\hline aip-miR162 & ath-miR162b & GW990392 & Arachis ipaensis & GCGATAAACCTCTGCATCCAG & -33.50 & 3 , & 1 & 95.1 & $90 \%$ & $3 e-18$ & 81 & 21 & 52 & 94 \\
\hline lja-miR162a & ath-miR162b & GO007141 & Lotus japonicus & TCGATAAACCTCTGCATCCAG & -33.60 & 3 , & 0 & 87.8 & $88 \%$ & $4 e-16$ & 80 & 21 & 47 & 88 \\
\hline lja-miR162b & ath-miR162b & GO015722 & Lotus japonicus & TCGATAAACCTCTGCATCCAG & -32.70 & 3 ' & 0 & 87.8 & $88 \%$ & $4 e-16$ & 80 & 21 & 47 & 90 \\
\hline 1lu-miR162 & ath-miR162b & BG149136 & Lupinus luteus & TCGATAAACCTCTGCATCCAG & -36.20 & $3^{\prime}$ & 0 & 141 & $90 \%$ & $1 \mathrm{e}-16$ & 79 & 21 & 47 & 82 \\
\hline psa-miR164 & ath-miR164a & FG530884 & Pisum sativum & TGGAGAAGCAGGGCACGTGCA & -34.20 & 5 , & 0 & 92.7 & $100 \%$ & $7 \mathrm{e}-07$ & 80 & 21 & 47 & 86 \\
\hline adu-miR166a & ath-miR166b & GW939731 & Arachis duranensis & TCGGACCAGGCTTCATTCCCC & -38.20 & $3{ }^{\prime}$ & 0 & 121 & $100 \%$ & $4 \mathrm{e}-04$ & 92 & 21 & 61 & 112 \\
\hline adu-miR166b & ath-miR166b & GW951206 & Arachis duranensis & TCGGACCACGCTTCATTC CCC & -38.10 & 3 , & 1 & 117 & $100 \%$ & 0.004 & 92 & 21 & 61 & 112 \\
\hline adu-miR166c & ath-miR166d & GW955263 & Arachis duranensis & TCGGACCAGGCTTCATTCCCC & -39.80 & 3 , & 0 & 124 & $100 \%$ & 0.015 & 96 & 21 & 61 & 71 \\
\hline ahy-miR166a & ath-miR166b & GO323023 & Arachis hypogaea & TCGGACCAGGCTTCATTCCCC & -36.60 & $3^{\prime}$ & 0 & 126 & $100 \%$ & $4 \mathrm{e}-04$ & 92 & 21 & 61 & 112 \\
\hline
\end{tabular}


Pure Appl. Bio., 2(3): 104-115, Sep- 2013.

\begin{tabular}{|c|c|c|c|c|c|c|c|c|c|c|c|c|c|c|}
\hline ahy-miR166b & ath-miR166b & GO330519 & Arachis hypogaea & TCGGACCAGGCTTCATTCCCC & -34.30 & 3 ' & 0 & 131 & $100 \%$ & $8 \mathrm{e}-06$ & 92 & 21 & 61 & 116 \\
\hline pvu-miR167 & ath-miR167b & FE692413 & Phaseolus vulgaris & TGAAGCTGCCAGCATGATCTA & -43.70 & 5 , & 0 & 95.1 & $99 \%$ & $3 e-18$ & 81 & 21 & 61 & 99 \\
\hline adu-miR168a & ath-miR168b & GW927363 & Arachis duranensis & TCGCTTGGTGCAGGTCGGGAA & -21.70 & 5 , & 0 & 99 & $60 \%$ & $2 \mathrm{e}-06$ & 92 & 21 & 61 & 60 \\
\hline adu-miR168b & ath-miR168b & GW945909 & Arachis duranensis & TCGCTTGGTGCAGGTCGGGCA & -52.00 & 5 , & 1 & 85.5 & $54 \%$ & 0.004 & 90 & 21 & 66 & 120 \\
\hline pvu-miR168 & ath-miR168b & FE674450 & Phaseolus vulgaris & TCGCTTGGTGCAGGTCGGGAA & -35.80 & 5 , & 0 & 98.2 & $57 \%$ & $8 \mathrm{e}-06$ & 94 & 21 & 61 & 140 \\
\hline adu-miR390 & ath-miR390a & GW943473 & Arachis duranensis & AAGCTCAGGAGGGATAGCGCC & -58.50 & 5 , & 0 & 114 & $71 \%$ & $2 \mathrm{e}-08$ & 97 & 21 & 57 & 126 \\
\hline aip-miR390 & ath-miR390a & GW966457 & Arachis ipaensis & AAACTCAGGAGGGATAGCGCC & -37.20 & 5 , & 1 & 110 & $94 \%$ & $4 e-10$ & 76 & 21 & 57 & 80 \\
\hline gur-miR393 & ath-miR393a & FS253174 & Glycyrrhiza uralensis & TCCAAAGGGATCGCATTGATCC & -52.60 & 5 , & 0 & 133 & $57 \%$ & $4 \mathrm{e}-04$ & 91 & 22 & 50 & 123 \\
\hline rps-miR394 & ath-miR394a & BI642560 & Robinia pseudoacacia & TTGGCATTCTGTCCACCTCC & -40.00 & 5 , & 0 & 92.7 & $62 \%$ & $4 \mathrm{e}-04$ & 87 & 20 & 55 & 109 \\
\hline lod-miR5630 & ath-miR5630 & GO319187 & Lathyrus odoratus & GCTGAGAGCGGTTCTGATGGC & -22.90 & 5 , & 2 & 80.6 & $100 \%$ & $6 e-14$ & 85 & 21 & 61 & 80 \\
\hline ahy-miR5658 & ath-miR5658 & GO330337 & Arachis hypogaea & ATGATGATGATGATGATGAAG & -12.30 & 5 , & 1 & 46.4 & $73 \%$ & 0.001 & 74 & 21 & 33 & 140 \\
\hline
\end{tabular}

Table \# 2: Comparison of Fabaceae, Ath and Li et al., reference values of GC content, core mfe, hairpin mfe and ch_ratio

\begin{tabular}{|c|c|c|c|c|}
\hline Reference & GC contents & Core mfe & Hairpin mfe & Ch_ratio \\
\hline Li et al. & $30-60(93 \%)$ & -42 to -17 (99\%) & -50.2 to -24.2 (99\%) & 50-96 (99\%) \\
\hline Ath & $36.4-51.1$ & -54.2 to -23.5 & -79.4 to -48.3 & $42-93$ \\
\hline Fabaceae & $42-61(93 \%)$ & -49.70 to $-17.2(72 \%)$ & -58.50 to $-31.90(83 \%)$ & $42-98(82.75 \%)$ \\
\hline
\end{tabular}


Fabaceae pre-miRNAs were subjected through BLAST against the protein database at NCBI using blastx and found no homology with known proteins. This result has confirmed our identified pre-miRNAs as strong candidates in Fabaceae. These findings are in agreement with the repeated works $[18,11,28,29]$.

\section{Sequence and Structural Features Filter}

It is an important step to ensure the pre-miRNAs from false positives. The filter is composed of four indices, namely GC content, core mfe, hairpin mfe and the ratio of core mfe to hairpin $\mathrm{mfe}$ (ch_ratio) (Barozai et al., 2008). As shown in (Table \# 2.), identified miRNAs of Fabaceae have a range of GC content $42-61 \%(93 \%)$, core mfe $(-49.70$ to $-17.2 \mathrm{kcal}$ $\mathrm{mol}^{-1}, 72 \%$ ), hairpin mfe -58.50 to $-31.90 \mathrm{kcal} \mathrm{mol}^{-1}$, $83 \%)$ ) and ch_ratio $(42-98,82.75 \%)$. The GC content and ch_ratio are within the range given by $\mathrm{Li}$ et al. [19] and Barozai et al. [11].

\section{Conservation and Phylogenetic Analysis of}

\section{Fabaceae miRNAs}

The conservation and phylogenetic analysis were made among the novel identified Fabaceae miRNAs with Arabidopsis lyrata (aly) and Brassica rapa (bra) (dicotyledonous), Brachypodium distachyon (bdi) and Triticum aestivum (tae) (monocotyledonous). The miRNAs 160 was considered with more conservation than others, as shown in (Figure\#2.) These findings were similar to Zhang et al. [18] and
Barozai et al. [11]. The conservation made them strong candidates of miRNAs in Fabaceae and suggests their conserved physiological function. The Phylogenetic tree showed that on the basis of premiRNA (miR-160) sequences, the Fabaceae is more closed to Triticum aestivum (tae), Brachypodium distachyon (bdi), Brassica rapa (bra) and Arabidopsis lyrata (aly) (Figure \# 3). The results are in agreement with the reported works $[\mathbf{1 1}, \mathbf{6}]$.

\section{The Fabaceae miRNAs Putative Targets}

The conserved nature of miRNAs in different organisms suggests their conserved function [7]. Total 61 targets were predicted for the 29 novel Fabaceae miRNAs, using a combination of BLAST and RNA-Hybrid algorithms (Table \# 3). Majority of these putative targets are already reported in different plant species $[7, \mathbf{1 1}, \mathbf{2 9}, \mathbf{3 0}]$. The Major portion of the predicted Fabaceae miRNAs targets are involved in transcription factor and metabolism $(40.98 \%, 26.22 \%)$ respectively, followed by signal transduction $(24.59 \%)$, transporter $(04.91 \%)$, growth and development (01.63\%) and (03.27\%) respectively.

Transcription factor (sequence-specific DNA-binding factor) is a key regulating factor that controls the transcription of genetic information from DNA to mRNA. Such targeted proteins were found in the newly identified miRNAs families (miR-156, 159, 160, 164, 166, 167 and 168) of Fabaceae in the 
Pure Appl. Bio., 2(3): 104-115, Sep- 2013.

current research work and the same results have been found by the other workers [6, 29].

Our findings were revealed that many miRNA families of Fabaceae take part in the regulation of metabolic path-way protein. These are; miR-160, miR-162, miR-164, miR-390, and miR-5630. These results were in agreement among the works of previously researchers $[31,29,12]$.

Experimental and computational studies have shown that miRNAs play a dynamic role in hormone signal transduction [32]. In our results $24.59 \%$ miRNAs were found to target Fabaceae genes that are involved in signal transduction. For instance miR-168, 393, 394, 5630 and 5658 target the signal transduction proteins like; F-box protein, Protein transport inhibitor response 1, Zinc finger $\mathrm{CCCH}$ domain-containing protein and PLTRP2E11 resistance protein PLTR gene.

In Fabaceae the miRNAs group, for instance miR390 and miR-5658 are involved in the transport pathway. These findings are incorporated with the earlier studies [10, 33].
Growth and development of an organism is regulated by gene functioning. The miRNAs are played a vital role in gene regulation during growth and development. Many miRNAs are involved in plant growth and development [17]. The putative Fabaceae miRNAs miR-167 and miR-168 respectively were found to target the proteins like; Putative pentatricopeptide repeat-containing protein and DNA repair protein of growth and development. These results have been found in the previous works $[7,34$, 35].

\section{Conclusions}

In current research work we adopted the similarity search methodology for the prediction of novel 29 miRNAs in 12 various Fabaceae species, using ESTs publically available. In addition, to identify their target proteins, we found different targeted mRNAs of the putative miRNAs families regarding transcription factors, signal transduction and growth and development. These newly identified miRNAs will be helpful in understanding various stages of the life cycle of Fabaceae plants. 


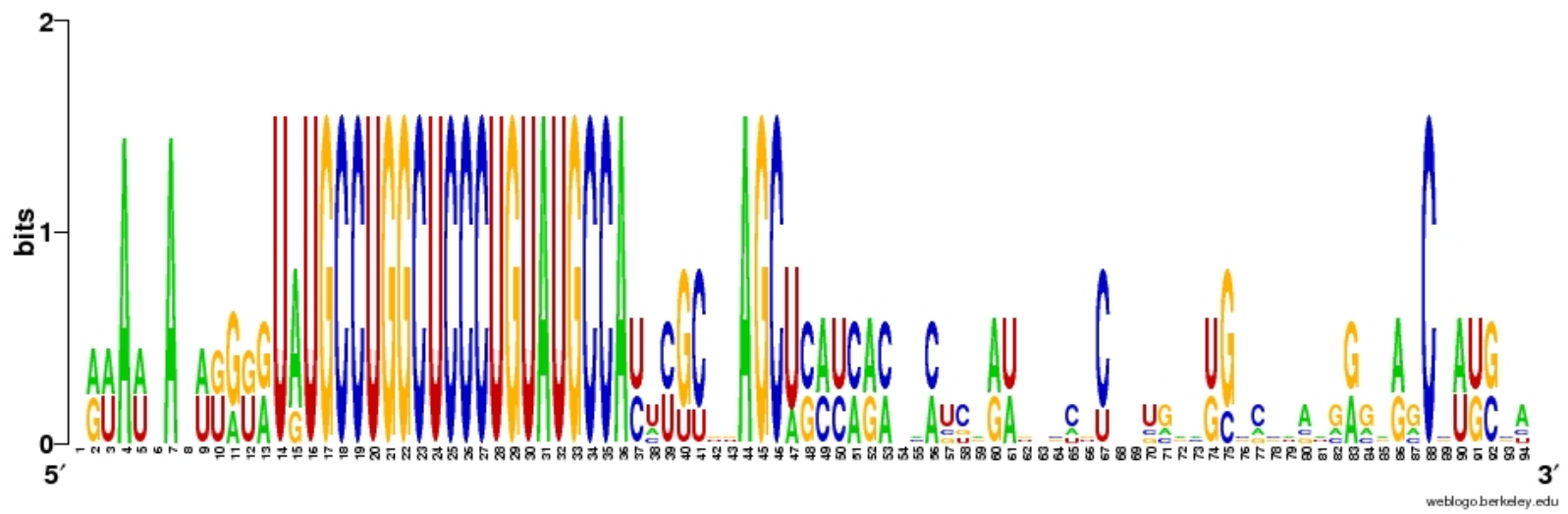

Figure \# 2: The Fabaceae miRNAs Conservation studies. Alignment of miRNAs (miR-160) of Fabaceae, (Lotus japonicus, lja) with Arabidopsis lyrata (aly), Brassica rapa (bra), Brachypodium distachyon (bdi) and Triticum aestivum (tae), using Weblogo, showing conserved nature of mature sequence highlighted in a rectangle box.

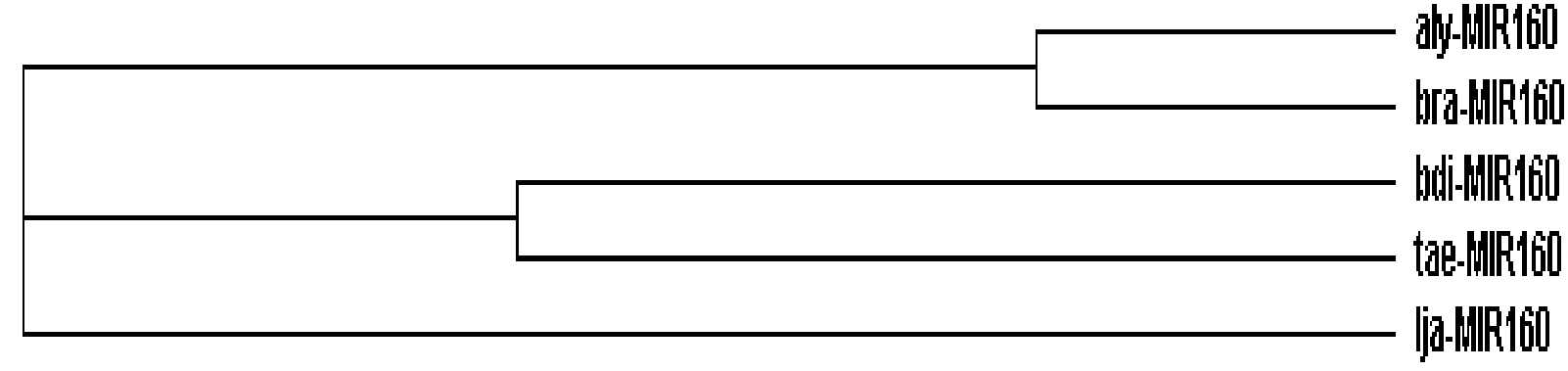

Figure \# 3: The Fabaceae miRNA phylogenetic analysis. The Phylogenetic analysis of the pre-miRNA (miR-160) of Fabaceae (Lotus japonicus, lja) with Arabidopsis lyrata (aly), Brassica rapa (bra), Brachypodium distachyon (bdi), Triticum aestivum(tae), is done with the help of Clustalw and cladogram tree was generated using neighbor joining clustering method. The Phylogenetic tree showed that on the basis of pre-miRNA sequences, the Fabaceae (Lotus japonicus, lja) is more closed to Triticum aestivum (tae), Brachypodium distachyon (bdi), Brassica rapa (bra) and Arabidopsis lyrata (aly). 


\section{References}

1. Bartel, D.P., 2004. MicroRNAs: Genomics, biogenesis, mechanism, and function. Cell. 116: 281297

2. Hunter, C. and R.S. Poethig, 2003. Missing links: miRNAs and plant development. Curr. Opin. Genet Dev. 13: 372-378

3. Carrington, J.C. and V. Ambros, 2003. Role of microRNAs in plant and animal development. Sci. 301: $336-338$

4. Lee R.C., Feinbaum R.L., Ambros V.C. 1993. The, elegans heterochronic gene lin-4 encodes small RNAs with antisense complementarity to lin-14, Cell $75 ; 843-854$

5. Berezikov, E., E. Cuppen and R.H. Plasterk, 2006. Approaches to microRNA discovery. Nat. Genet. 38 (Suppl): 2-7

6. Barozai, M.Y.K., I.A. Baloch and M. Din, 2011 b. Identification of MicroRNAs and their targets in Helianthus. Mol Biol Rep. 39: 2523-2532

7. Aukeman, M.J. and H. Sakai, 2003. Regulation of flowering time and floral organ identity by a microRNA and its APETALA2-like target genes. Plant Cell. 15: 2730-2741

8. Kurihara, Y. and Y. Watanabe, 2004. Arabidopsis thaliana micro-RNA biogenesis through Dicer-like 1 protein functions. Proc. Natl. Acad. Sci. USA. 101: 12753-12758

9. Mallory, A.C. and H. Vaucheret, 2006. Functions of microRNAs and related small RNAs in plants. Nat. Genet. 38: 31-36

10. Sunkar, R. and J.K. Zhu, 2004. Novel and stressregulated microRNAs and other small RNAs from Arabidopsis thaliana. Plant Cell. 16: 2001-2019

11. Barozai, M.Y.K., M. Irfan, R. Yousaf, et al., 2008. Identification of micro-RNAs in cotton. Plant Physiol. Biochem. 46(8-9): 739-751
12. Barozai, M.Y.K., I.A. Baloch and M. Din, 2011c. Computational identification of microRNAs and their targets in two species of evergreen spruce tree (Picea). PWASET. 75: 413-418

13. Griffiths-Jones, S. 2004. The microRNA Registry. Nucleic Acids Res. 32(D): 109-111

14. Altschul, S.F., W. Gish, W. Miller, E.W. Myers and D.J. Lipman, 1990. Basic local alignment search tool. J. Mol. Biol. 215: 403-410

15. Stephen, F.A., T.L. Madden, A.A. Schaffer, et al., 1997. Gapped BLAST and PSI-BLAST, new generations of protein database search programs. Nucleic Acids Res. 25: 3389-3402

16. Zuker, M. 2003. Mfold web server for nucleic acid folding and hybridization prediction. Nucleic Acids Res. 31: 3406-3415

17. Barozai, M.Y.K., M. Din and I.A. Baloch, 2011a. Identification of microRNAs in ecological model plant Mimulus. J. Biophys. Chem. 2(3): 322-331

18. Zhang, B., X. Pan, C.H. Cannon, G.P. Cobb and T.A. Anderson, 2006. Conservation and divergence of plant microRNA genes. Plant J. 46: 243-259

19. Li, S.C., C.U. Pan and W.C. Lin, 2006. Bioinformatics discovery of microRNA precursors from human ESTs and introns. BMC Genomics. 7: 164-174

20. Crooks, G.E., G. Hon, J.M. Chandonia and S.E. Brenner, 2004. Web-Logo: a sequence logo generator. Genome Res. 14: 1188-1190

21. Larkin, M.A., G. Blackshields, N.P. Brown, R. Chenna, et al., 2007. ClustalW and ClustalX version 2. Bioinform. 23: 2947-2948

22. Barozai, M.Y.K., 2012a. Identification and characterization of the microRNAs and their targets in Salmo salar. Gene 499:( 1) 163-168 
23. Kruger, J. and M. Rehmsmeier, 2006. RNA hybrid: microRNA target prediction easy, fast and flexible. Nucleic Acids Res. 34(Suppl 2): 451-454

24. Barozai, M.Y.K. and T. Husnain, 2011. Identification of biotic and abiotic stresses upregulated ESTs in Gossypium arboreum. Mol Biol Rep. 39(2): 1011-1018

25. Barozai, M.Y.K. and A.H. Wahid, 2012. Insilico identification and characterization of cumulative abiotic stress responding genes in Potato (Solanum tuberosum L.). Pak. J. Bot. 44: 57-69

26. Ambros, V., B. Bartel and D.P. Bartel, 2003. A uniform system for microRNA annotation. RNA. 9: 277-279

27. Reinhart, B.J., E.G. Weinstein, M.W. Rhoades, B. Bartel and D.P. Bartel, 2002. MicroRNAs in plants. Genes Dev. 16: 1616-1626

28. Sunker, R. and Jagdeeswaran, G. 2008. In-silico identification of conserved microRNAs in large number of diverse plant species. BMC Plant Biol. 8: 37

29. Fuliang, X., P.F. Taylor and B. Zhang, 2010. Identification and characterization of microRNAs and their targets in the bioenergy plant switchgrass (Panicum virgatum). Planta. 232: 417-434
30. Xue-fei, D., N. Cui, L. Wang, X. Zhao, B. Qu, et al., 2012. The SnRK Protein Kinase Family and the Function of SnRK1 Protein Kinase. Int. J. Agric. Biol., 14 (4): 575-579

31. Xie, F.L., S.Q. Huangm, K. Guo, A.L. Xiang, Y.Y. Zhu, L, Nie and Z.M. Yang, 2007. Computational identification of novel microRNAs and targets in Brassica napus. FEBS Lett. 581: 14641474

32. Jia, X., W.X. Wang, L. Ren, Q.J. Chen, V. Mendu, B. Willcut, R. Dinkins, X. Tang and G. Tang, 2009. Differential and dynamic regulation of miR398 in response to $\mathrm{ABA}$ and salt stress in Populus tremula and Arabidopsis thaliana. Plant Mol. Biol. 71: $51-59$

33. Jones-Rhoades, M.J. and D.P. Bartel, 2004. Computational identification of plant microRNAs and their targets, including a stress induced miRNA. Mol. Cell. 14: 787-799

34. Chen, X., 2004. A microRNA as a translational repressor of APETALA2 in Arabidopsis thaliana flower development. Sci. 303: 2022-2025

35. Barozai, M.Y.K., 2012b. The novel 172 sheep (Ovis aries ) microRNAs and their targets. Mol Biol Rep. 39(5):6259-6266 